\title{
Technological change and fisheries sustainability: The point of view of adaptive dynamics
}

Fabio Dercole (fabio.dercole@polimi.it) Charlotte Prieu (prieu@biologie.ens.fr) Sergio Rinaldi (rinaldi@elet.polimi.it)

\section{Approved by}

Ulf Dieckmann

Program Leader, EEP

June 2011 Institute, its National Member Organizations, or other organizations supporting the work. 


\section{Contents}

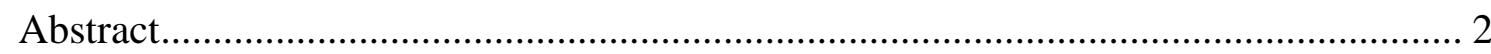

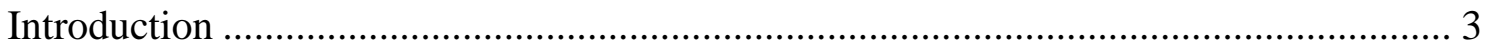

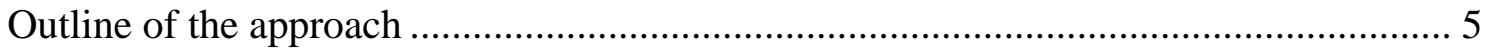

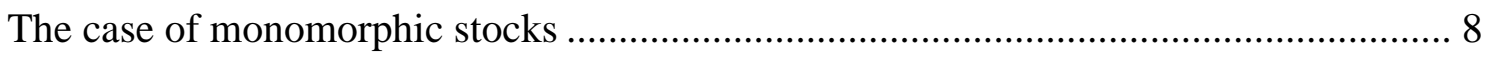

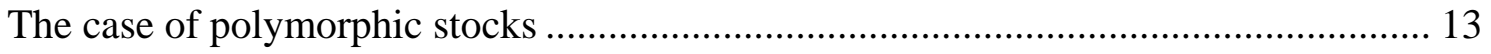

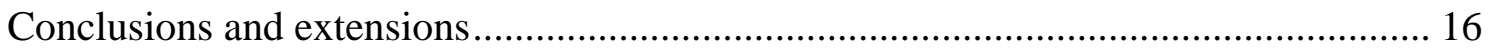

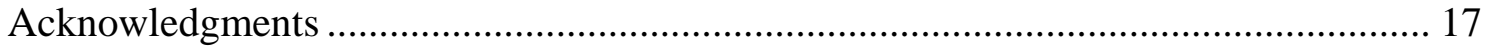

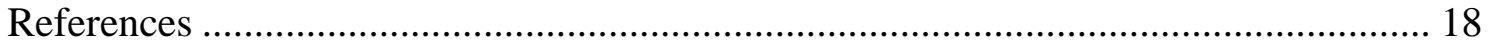

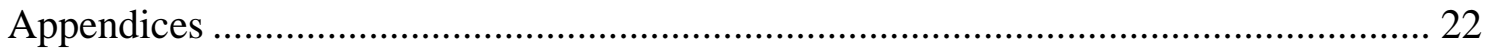

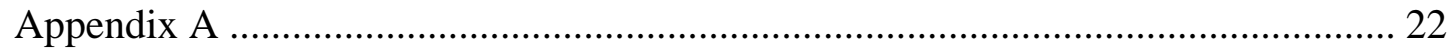

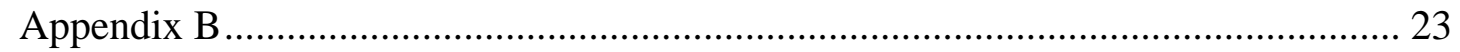

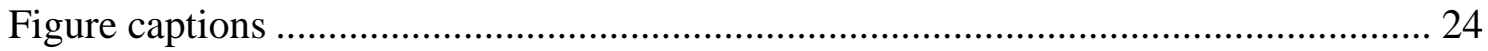

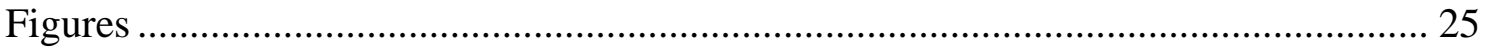




\title{
Technological change and fisheries sustainability: The point of view of Adaptive Dynamics
}

\author{
Fabio Dercole ${ }^{1,4}$, Charlotte Prieu ${ }^{2}$, and Sergio Rinaldi ${ }^{1,3}$
}

${ }^{1}$ DEI, Politecnico di Milano, Italy

${ }^{2}$ Biology Department, Ecole Normale Supérieure, Paris, France

${ }^{3}$ EEP, International Institute for Applied Systems Analysis, Laxenburg, Austria

${ }^{4}$ To whom correspondence should be addressed

Address: Via Ponzio 34/5, 20133 Milano, Italy

Ph: +3902 2399 3484; Fax: +3902 23993412

E-mail: fabio.dercoleepolimi.it 


\section{Abstract}

The analysis of a simple model shows that exploitation of fish stocks can entrain in the long run the substantial decline or even the collapse of the stock, as well as difficulties in stock recovery, loss of fishery resilience, and reduction of the mean fish size. The results are in agreement with numerous observations, even though they are obtained with a simple model in which the harvesting fleet and the fish stock are considered as unstructured predator and prey. The study is carried out for the typical case of fleet dimension not too sensitive to the year-to-year fluctuations of the stock and assuming that the sole cause of evolution is technological innovation. The analysis is performed by means of Adaptive Dynamics, an approach born in theoretical biology which is used here in the context of technological change. Although the results are qualitatively consistent with those obtained long ago through the principles of bioeconomics, it is fair to stress that the underlying assumptions are different. In fact, in the bioeconomic approach fleet technology does not evolve and fishing effort varies to produce economic optimization, while in the Adaptive Dynamics approach technological innovation is the key driver. The paper is purely theoretical and the proposed model can hardly be tuned on any real fishery. No practical guidelines for managers can therefore be drawn, if not the general conclusion that long-term sustainability of exploited fish stocks can only be achieved if strategic parameters influencing technological change are kept under strict control.

Key words: Adaptive Dynamics, bifurcation analysis, collapse of fish stocks, fish body size, fishery resilience, technological innovation. 


\section{Introduction}

2 The history of commercial fisheries reveals that many, if not all, exploited fish stocks enter, sooner or 3 later, a phase of deep crisis. In particular, the available data (see, for example, the Ransom Myers' Stock 4 Recruitment Database) point out the following five general facts.

(i) Stock abundances decline over time. Perhaps the best known example is the case of Atlantic cod, a species that supported one of the largest and economically most important fisheries throughout the North Atlantic for centuries, and declined more than 90\%. But the problem is very general, as ascertained by Hutchings and Reynolds (2004), who have studied 230 populations and found a median reduction of $83 \%$ in breeding populations size from known historic levels.

(ii) Stocks can collapse. The continuous decline of stock abundances is often exacerbated during short periods of time (years, decades) and leads to very low abundances or to extinction. This has obvious social and economic implications, but also profound indirect ecosystem effects involving the entire food chain (Jackson et al., 2001; Bellwood et al., 2004; Hutchings and Reynolds, 2004; Scheffer et al., 2005).

(iii) Recovery after depletion is slow. Historical data indicate that the recovery of a stock after severe depletion is often very slow and not always guaranteed. The analysis suggests that recovery is related to fishing, taxonomic affiliation, habitat, and life history, with fishing being the dominant factor (Hutchings and Reynolds, 2004).

(iv) Fisheries become fragile. The dynamics of harvested stocks depend upon available resources and fishing pressure that both evolve over time. Limited though significant evidence (Anderson et al., 2008) indicates that stocks gradually become less resilient when fishing pressure increases. This means that small demographic fluctuations and/or small accidental environmental perturbations can easily trigger a temporary depletion of the stock, followed by a slow recovery.

(v) Body size decreases. The best example is again that of Atlantic cod (see, however, Jørgensen et al., 2007, Table S1, and Hutchings and Reynolds, 2008, Table 1, for other species), for which archaeological records and recent fishery data in the coastal Gulf of Maine show an impressive reduction of body length (from 100 to $20 \mathrm{~cm}$ today, see Fig. 2 in Jackson et al., 2001). More precisely, a limited 
decay, over 5000 years, certainly associated with the long-term adaptation to harvesting and other selective pressures driven by genetic mutations, is followed by a more relevant decay occurred in the last decades, i.e., at demographic timescale, and most likely due to a strong harvesting pressure reshaping the size distribution of the stock.

Moreover, specifically organized field surveys and data analysis (Hilborn and Walters, 1992; Barot et al., 2004a,b; Olsen et al., 2004) focused on the age, stage, and size structures of exploited fish stocks, and showed remarkable trends of related life-history traits (in particular, the so-called maturation reaction norm). Here, however, we show that facts (i)-(v) can be derived without making explicit reference to the structural properties describing the life course of individuals. We therefore consider a simple model with unstructured fish populations, i.e., composed of identical (adult) individuals, and do not discuss structurally-specific empirical observations.

Properties (i)-(v) may result from various mechanisms. Long-term response of ecosystems to climatic variations is the first that comes to mind. However, this mechanism would not explain why stocks have a systematic and perverse tendency to deteriorate over time. It is therefore obvious to conjecture that the selective pressure induced by harvesting is the real key factor. Selective pressures control the long-term phenotypic evolution of the stock driven by genetic mutations, as well as the technological change of the fleet driven by innovation processes. This naturally calls for studies in which the two compartments of the fishery (the stock and the fleet) are characterized by coevolving (biological and technological) traits. Of course, the analysis of the two extreme cases with models in which either technological innovations or genetic mutations are inhibited is more simple. This is why the studies carried out so far (see, e.g., Ernande et al., 2004; de Roos et al., 2006) refer to models in which the technological level of the fleet is assumed constant (see Heino, 1998, for a naive exception, where the fishing strategy is updated only after biological traits halt at evolutionary equilibria). Here we follow the opposite option, by focusing on a timescale (years, decades) on which genetic mutations can be neglected, while technological innovations play a significant role. This is justified by the impressive improvement of the fishing technology that occurred in the last decades (Salthaug, 2001; Hannesson, 2002; Walters and Martell, 2004).

The model of the fishery we consider is a so-called minimal model: it is fully deterministic (i.e., there are no sources of uncertainty) and both the stock and the fleet have no explicit structure describing the life course of individuals (fish and boats). Moreover, the model is not specific on a number of significant details 
characterizing real fisheries, such as the species under exploitation, the geographic location, the fishing gears, and the management policy. The model is therefore not very tight to reality and cannot be used to draw quantitative predictions. Nevertheless, it is interesting because, being abstract, it confirms that the five facts listed above are indeed general.

The analysis is performed through the Adaptive Dynamics (AD) approach (Metz et al., 1996; Geritz et al., 1997, 1998) and, more precisely, through the so-called AD canonical equation (Dieckmann and Law, 1996, see also the recent book by Dercole and Rinaldi, 2008) that describes the long-term evolution of an adaptive trait by means of an ordinary differential equation (ODE). The approach finds its origin in the study of mutation and selection processes in biology and considers rare mutations of small effects. The novelty, here, is that the AD approach is used to describe the evolution of the technological level of the fleet. In other words, existing boats in the fleet compete with innovative ones, resulting in a continuous evolution of the underlying technological characteristics of successful boats.

\section{Outline of the approach}

Technological change is the result of innovation and competition processes (Ziman, 2000). In all context (biological, social, economic,...) an innovation is a change, in one or more of the features characterizing the interacting agents of the evolving system, with respect to the current composition of the system. In the technological context, innovations are associated with changes in the technological level of the involved products. If the technological level of a product is identified with, e.g., the number of its technological components (or, more in general, with the sum of suitable weights associated to each component), all innovations corresponding to the simple addition of an extra component (the most common case in practice) are associated with an increase of technological level. However, the addition of an extra component which entrains the elimination of other components can also lead to innovative products with lower technological content. Moreover, new products obtained from the present ones by simply eliminating one or more components are also innovative (by definition), though such kind of innovations are not associated with any technological novelty and can give rise to technological solutions already adopted in the past.

We imagine that, in the absence of technological change, boats are all identical and that technological innovations appears from time to time, so that just after an innovation the fleet has two components: $B$ so-called resident boats with a technological level $x$ and $B^{\prime}$ innovative boats with the technological level 
$x^{\prime}$. By contrast, no phenotypic mutations occur in the fish stock. Under these assumptions, the short-term interactions between the stock $F$ and the two components of the fleet $B$ and $B^{\prime}$ are described by three ODEs of the form

$$
\begin{aligned}
\dot{F} & =f\left(F, B, B^{\prime}, x, x^{\prime}\right), \\
\dot{B} & =B g\left(F, B, B^{\prime}, x, x^{\prime}\right), \\
\dot{B}^{\prime} & =B^{\prime} g\left(F, B^{\prime}, B, x^{\prime}, x\right)
\end{aligned}
$$

(see next section for details), where $F$ is a scalar if the stock is monomorphic or a vector if the stock is polymorphic $\left(F_{i}, i=1, \ldots, N\right.$ are the abundances of each morph). Model (1) is a prey-predator model with one prey $(F)$ and two predators $\left(B\right.$ and $\left.B^{\prime}\right)$ competing for the same resource. If $x^{\prime}=x$, all boats are identical, so that the fleet is homogeneous and its short-term interactions with the stock are described by

$$
\begin{aligned}
& \dot{F}=f(F, B, 0, x, x), \\
& \dot{B}=B g(F, B, 0, x, x),
\end{aligned}
$$

where $B$ is the total number of boats of the fleet. We assume that in large regions of parameter space, model (2) has a unique stable equilibrium $(\bar{F}(x), \bar{B}(x))$, as is the case for the models described in Sects. 3 and 4. If two slightly different technologies $x$ and $x^{\prime}$ are both associated with stable equilibria, the principle of competitive exclusion predicts that one of the two technologies prevails on the other, so that the final outcome is a fleet with a unique technological level. That is to say, $x$ remains unchanged if the innovative trait looses the competition, while in the opposite case $x$ is substituted by $x^{\prime}$. If innovations are sufficiently rare to guarantee that the substitutions $x \rightarrow x^{\prime}$ are fully realized, the technological level varies through a series of small steps. Under suitable hypothesis on the innovation process, the dynamics of the (expected) technological level are described, on a longer, say evolutionary, timescale, by the following ODE:

$$
\dot{x}=\left.\frac{1}{2} \mu \sigma^{2} \bar{B}(x) \frac{\partial}{\partial x^{\prime}} g\left(\bar{F}(x), 0, \bar{B}(x), x^{\prime}, x\right)\right|_{x^{\prime}=x}
$$

where $\mu$ and $\sigma^{2}$ measure the frequency and variance of the innovations and $g\left(\bar{F}(x), 0, \bar{B}(x), x^{\prime}, x\right)$ is the initial growth rate (per boat) of the innovative component of the fleet. Equation (3) is the so-called AD 
canonical equation (Dieckmann and Law, 1996; Dercole and Rinaldi, 2008). It is derived from the short-term model (1) and from the statistics $\mu$ and $\sigma^{2}$ of the innovation process, and predicts the long-term dynamics of the technological level $x$.

By contrast, in the case of a radical innovation (a significant novelty, or a relevant dismission of technologies considered ineffective and/or too costly), namely when $x$ and $x^{\prime}$ are remarkably different, the outcome of the competition must be established by means of model (1). Once the new equilibrium has been determined, the AD canonical equation describes the next smooth phase driven by innovations of small effects. In other words, $\mathrm{AD}$ describes technological change as a continuous process driven by marginal innovations punctuated, from time to time, by major breakthroughs. Note that radical innovations can penetrate without substituting the resident technology: the innovation can be only temporary but imply the switch of the resident technology to a new equilibrium; or the two technologies, $x$ and $x^{\prime}$ can coexist, with the consequent diversification of the fleet; or both technologies can disappear, marking the end of the fishery. The outcome of the competition depends on the global structure of model (1) and cannot be a priori predicted.

Interestingly, $\mathrm{AD}$ shows that the above phenomena can be triggered also by marginal innovations. In fact, when the evolution of $x$ slows down while approaching a value $\bar{x}$ annihilating the right-hand side of (3), a deeper investigation (Geritz et al., 1997, 1998; Dercole and Rinaldi, 2008) shows that a branching phenomenon can occur: a small innovation gives rise to a new component of the fleet which does not have the power of outcompeting the old one but at the same time is not outcompeted. Moreover, subsequent innovations in the two coexisting components lead to their technological diversification. This means that once the technological level $\bar{x}$ is reached (or almost reached), it is possible that the fleet splits into two different fleets, that are initially very similar but then diversify. In particular, one should expect that branching could more easily occur when the stock is polymorphic, because in such a case the existence of different fleets exploiting the characteristics of different components of the stock would not be in contrast with the principle of competitive exclusion. The branching conditions are not reported here, because they are not used in the discussion that follows.

While if $x$ varies in accordance with (3) and reaches in finite time a value $x^{*}$ at which the equilibrium $(\bar{F}(x), \bar{B}(x))$ looses stability, i.e., a bifurcation of the resident model (2), a totally different phenomenon occurs. In fact, when the technological level $x$ approaches $x^{*}$ the fishery becomes less resilient, in the sense that small demographic fluctuations around the equilibrium and/or small environmental perturbations can have relevant consequences for a very long time. Moreover, as shown in the next section, the low 
sensitivity of the fleet dimension $\left(B+B^{\prime}\right)$ to the high-frequency (year-to-year) fluctuations of the fish stock (due to many socio-economic factors, like the public or private support to fisheries and the typical inertia of fishermen in giving up with their job when stocks are scarce) can easily produce very long periods of low abundance which can hardly be distinguished from stock collapses.

\section{The case of monomorphic stocks}

Although fish stocks are always characterized by a certain degree of genetic diversity, we analyze in this section the extreme case of monomorphic stocks. This simplifies the model and minimizes the computational effort needed to derive the first four properties discussed in the Introduction.

The specific model (1) on which we focus from now on is a variant of the most standard prey-predator model (Rosenzweig and MacArthur, 1963), which is here adapted to the case of managed fisheries. The equations are

$$
\begin{aligned}
\dot{F} & =(b-d) F-\gamma_{0} F^{2}-E(F) H\left(F, B, B^{\prime}, x\right) B-E(F) H\left(F, B^{\prime}, B, x^{\prime}\right) B^{\prime}, \\
\dot{B} & =\frac{r}{v(x)}\left[p w E(F) H\left(F, B, B^{\prime}, x\right)-c_{0}\left(1-\frac{\delta_{e}\left(B+B^{\prime}\right)}{B_{e}+B+B^{\prime}}\right)\right] B-D B, \\
\dot{B}^{\prime} & =\frac{r}{v\left(x^{\prime}\right)}\left[p w E(F) H\left(F, B^{\prime}, B, x^{\prime}\right)-c_{0}\left(1-\frac{\delta_{e}\left(B^{\prime}+B\right)}{B_{e}+B^{\prime}+B}\right)\right] B^{\prime}-D B^{\prime},
\end{aligned}
$$

where

$$
H\left(F, B, B^{\prime}, x\right)=\frac{a(x) F^{2}}{1+a(x) h(x) F^{2}-\delta_{c}\left(B+B^{\prime}\right) /\left(B_{c}+B+B^{\prime}\right)}
$$

is the so-called functional response, namely the harvest rate per boat, while all other new symbols represent positive demographic, environmental, and economic parameters, some of which are assumed to depend upon the technological levels $x$ and $x^{\prime}$ of the resident and innovative boats. In order of appearance: $b$ and $d$ are basal birth and death rate of the fish population and $\gamma_{0}$ measures intraspecific competition, so that $K=(b-d) / \gamma_{0}$ is the carrying capacity of the unexploited stock; $E(F)$ describes the exploitation policy, which aims at preventing fishing if the stock is below a threshold abundance $F_{0}$ (see the first equation below and the related comment); $r$ is the fraction of the net income (the term within brackets in eqs. (4b) and (4c)) which is reinvested into new resident and innovative boats of value $v(x)$ and $v\left(x^{\prime}\right) ; p$ is the price (per unit weight) at which all catches are sold and $w$ is the fish body weight; $c_{0}$ is the maintenance cost of a single boat 
in the absence of economies of scale, that are taken into account by a discount $\delta_{e}$ realized (with sensitivity measured by $\left.1 / B_{e}\right)$ when the fleet dimension $\left(B+B^{\prime}\right)$ is large; $1 / D$ is the (average) life time of a boat; $a(x)$ is the harvest attack rate in the absence of cooperation and coordination among the boats of the fleet, namely $a(x) F^{2}$ is the harvest rate realized when both the stock and the fleet are scarce; if no distinction is made between resident and innovative boats, and the major effect of cooperation is to reduce the time needed to locate the stock by a factor at most equal to $\delta_{c}$ when the fleet is large, then the realized attack rate is $a(x) /\left(1-\delta_{c}\left(B+B^{\prime}\right) /\left(B_{c}+B+B^{\prime}\right)\right)$, where $1 / B_{c}$ controls the sensitivity of the searching time with respect to the fleet dimension; $h(x)$ is the handling time per fish, responsible of the saturation of the functional response.

As for the dependence upon the resident and innovative technological levels $x$ and $x^{\prime}$, many are the possible choices. Here we like to make general assumptions, without referring to a particular species and/or fishery, and limit both the number of parameters influenced by the technology and the number of (second level) parameters which control such influences. Our assumptions are listed below.

- The value of the boat $v(x)$ increases with $x$ more than linearly, starting from a reference value $v_{0}$ corresponding to the technology $x=0$ in use at the beginning of the exploitation. This sets a price to be paid to be technologically more advanced and technically avoids unrealistically unbounded results.

- The attack rate $a(x)$ increases with $x$, capturing the higher harvesting power of a more technologically advanced boat, but saturates for large $x$, describing the fact that no technology can realize extremely aggressive harvesting rates.

- In line with the last choice, the handling time $h(x)$ is decreasing (and saturating) with $x$, capturing the technological improvements in the handling and/or transportation of the catch.

- The specific functions we use in the numerical analysis are the following:

$$
\begin{aligned}
E(F) & =\frac{F^{e}}{F_{0}^{e}+F^{e}}, \quad e>1, \\
v(x) & =v_{0}\left(1+\left(x / x_{v}\right)^{v_{1}}\right), \quad v_{1}>1, \\
a(x) & =a_{0}\left(1+\frac{\delta_{a} x / x_{a}}{1+x / x_{a}}\right) \\
h(x) & =h_{0}\left(1-\frac{\delta_{h} x / x_{h}}{1+x / x_{h}}\right) .
\end{aligned}
$$


Note that, strictly speaking, function $E(F)$ does not prevent fishing when $F<F_{0}$, but well-approximates the discontinuous function $E(F)=0$ for $F<F_{0}, E(F)=1$ for $F \geq F_{0}$, if $e$ is sufficiently large ( $e=4$ in all our computations). The choice of a smooth (though sharp) $E(F)$ is obligate if one wants to rely on the standard methods for bifurcation analysis.

In principle, all parameters should be fixed at different values for tuning the model on different fleets and stocks. Here we do not constrain the parameters since, as shown below, our results are definitely robust with respect to parameter variations. There is, however, one exception, namely that the two parameters $r / v_{0}$ and $D$ entering the fleet equation must be small. This constraint is introduced in order to guarantee that the fleet dimension varies slowly in time in comparison with the stock abundance, as a consequence of the frequent non existence of alternative jobs for fishermen and the use of subsidies in economically stressed fisheries. The constraint takes also into account that depleted species continue to be caught as bycatch in other fisheries (Alverson et al., 1994) and that closed fisheries tend to be reopened at the first sign of population increase (Hutchings and Reynolds, 2004).

Model (4) contains four major differences with respect to the standard Rosenzweig-MacArthur model: the exploitation policy $E(F)$ (equal to 1 in the standard model) that can be varied by a fishing agency, the type of the functional response (III instead of II) that takes into account that fish have safe niches where the boats cannot operate, the cooperation that is particularly important in those fisheries where fishing efficiency is enhanced when properly coordinated, and the economies of scale that are relevant in fisheries where boat maintenance, catch handling, and transportation of the terminal products are globally shared. In the following we present the results obtained with model (4) because the phenomena justifying the four variants are often present in commercial fisheries. However, the results remain qualitatively the same even if some or all of the variants are ignored.

We are now at the point of writing the $\mathrm{AD}$ canonical equation (3) but, unfortunately, the equilibrium $(\bar{F}(x), \bar{B}(x))$ cannot be computed explicitly. This is not a serious obstacle, because, as shown in Appendix A, it is possible to associate to the differential equation (3) a set of two algebraic relationships defining the equilibrium and then solve the differential-algebraic system of equations to compute the evolution of the technological level $x$.

As described in the previous section, it is also necessary to perform a bifurcation analysis of the resident model (2), at least with respect to parameter $x$. This can be effectively done by using continuation methods 
(see, e.g., Allgower and Georg, 1990, or Kuznetsov, 2004) and software oriented to the bifurcation analysis of dynamical systems (here we have used MATCONT, see Dhooge et al., 2002). This has already been done for prey-predator models similar to model (4) (Muratori and Rinaldi, 1989; Kuznetsov et al., 1995; Rinaldi and Gragnani, 2004) and is therefore not reported here. Figure 1 shows the state portraits of model (2) for two slightly different values of $x$, one smaller and one greater than a bifurcation value $x^{*}$. Before the bifurcation (Fig. 1a) there is a unique stable equilibrium $(\bar{F}, \bar{B})$ and perturbed trajectories (see the trajectory starting from point 1) can go very far from the equilibrium before returning to it. This feature is not due to the particular parameter setting used to produce Fig. 1 but is rather generic, since it is a consequence of the slow-fast nature of the system (see Rinaldi and Scheffer, 2000). In fact, since the fleet dimension can vary only very slowly, the stock can decrease quite consistently in a relatively short time while the boats remain practically constant (segment 1-2 of the trajectory); then the stock can remain scarce for a very long time during which the boats slowly abandon the fishery (segment 2-3). Thus, before the bifurcation, the fishery has low resilience, since even small perturbations from the equilibrium (from $(\bar{F}, \bar{B})$ to point 1) can give rise to very long transients perceived as collapses of the stock. After the bifurcation (Fig. 1b) the equilibrium $(\bar{F}, \bar{B})$ is unstable and trajectories tend toward a limit cycle which, in the case of slow-fast systems, is very large and characterized by a fast collapse of the stock (segment 1-2) followed by a long phase of slow decay of the fleet (segment 2-3).

The bifurcation described in Fig. 1 is known as Hopf bifurcation and describes the birth of a limit cycle associated with the change of stability of an equilibrium (see, e.g., Strogatz, 1994, or Kuznetsov, 2004). Model (2) has many other bifurcations (see Kuznetsov et al., 1995, for a similar case). In particular, the so-called transcritical bifurcation characterizes the collision of the equilibrium $(\bar{F}, \bar{B})$ with the equilibrium $(K, 0)$. The two equilibria exchange stability through the bifurcation, so that after the bifurcation the trajectories of model (2) tend toward $(K, 0)$, i.e., the fleet goes extinct because economically unsustainable and the stock remains unexploited.

Other bifurcations involve the limit cycle, but they are of no practical interest. The reason is that the dynamics predicted by model (2) after the collapse of the stock and the consequent decay of the fleet (i.e., after point 3 in both cases of Fig. 1) cannot be interpreted in terms of the real fishery. The model simply predicts the end of the fishery. Whether the fish stock will recover and the same or a new fishery will start in the future is beyond the scope of the model predictions. In particular, Fig. 1b should not be interpreted as a cyclic predator-prey chase between the harvesting fleet and the exploited stock. For this reason, in the 
following we consider only technological levels $x$ for which the equilibrium $(\bar{F}, \bar{B})$ is stable, and we focus only on its relevant bifurcations, namely the Hopf and the transcritical.

We have systematically performed a two-dimensional bifurcation analysis with respect to $x$ and any other parameter, say $q_{i}$, of model (2), thus obtaining a Hopf and a transcritical bifurcation curve in each plane $\left(q_{i}, x\right)$. In the region between the two curves, the equilibrium $(\bar{F}, \bar{B})$ is positive and stable, so that technological change takes place. In this region we have also drawn the constant solution $\bar{x}\left(q_{i}\right)$ of the canonical equation (3). The typical result is shown in Fig. 2 for two strategic parameters $q_{i}$, namely the attack rate $a_{0}$ and the protectionism threshold $F_{0}$. The figure points out a number of general and interesting properties. First, the sign of $\dot{x}$ in (3) is positive at low values of the technological level $x$ and negative at high values. This means that fisheries starting with poor primitive technologies can only improve their technological level, as indeed it occurred historically. Of course, the negative trend of technological change represents fisheries starting from too high technological levels. Second, if the attack rate is sufficiently low or the protectionism is sufficiently high, the technological level of the fleet tends toward the equilibrium $\bar{x}\left(q_{i}\right)$, which, however, is associated to an equilibrium $(\bar{F}, \bar{B})$ with low resilience if point $\bar{x}\left(q_{i}\right)$ is close to the Hopf bifurcation curve. By contrast, if the attack rate is high or protectionism is low, the technological level $x$ evolves toward the value $x^{*}$ at which stock and fleet in principle start oscillating with large amplitudes and, in practice, collapse. Thus, the final message is that it is rather difficult to guarantee the sustainability of a commercial fishery unless strategic control parameters, like attack rate and protectionism, are kept at very safe levels.

Two other properties also emerge from our analysis. The first is that technological change can easily force a fishery to approach the edge of its most complex dynamic behavior (see Fig. 2). This fact seems to complement earlier findings about the possibility that mutation and selection processes force ecosystems to evolve toward the edge of chaos (Ferrière and Gatto, 1993; Ellner and Turchin, 1995; Dercole and Rinaldi, 2008). The second is essentially a mathematical curiosity, namely the fact that the curve $\bar{x}\left(q_{i}\right)$ of the equilibria of the canonical equation (see Fig. 2) starts from the extreme point of the Hopf bifurcation curve and depends only very weakly upon the parameter $q_{i}$. Although this has only been observed numerically, it implies that technological change always erodes the fishery resilience, possibly up to its collapse, by driving model (2) close to the Hopf bifurcation.

Up to now, we have shown properties (ii), (iii), and (iv) mentioned in the Introduction (property (v) makes no sense in the case of monomorphic stocks) so that it only remains to check that technological 
change implies the decline of the stock abundance. This has been done by systematically drawing in any two-dimensional space $\left(q_{i}, x\right)$ the curves $\bar{F}\left(q_{i}, x\right)=$ const. The result, reported in Fig. 3 for the two cases already described in Fig. 2, shows indeed that the technological change predicted by the AD canonical equation (3) (see vertical trajectories in Fig. 2) is associated with a decline of the stock abundance $\bar{F}$.

It is interesting to note that the yield $\bar{Y}$ of the fishery at equilibrium is given by (see eq. 4a)

$$
\bar{Y}=(b-d) \bar{F}-\gamma_{0} \bar{F}^{2},
$$

so that the curves at constant abundance in the space $\left(q_{i}, x\right)$ are also curves at constant yield. Moreover,

$$
\frac{\partial \bar{Y}}{\partial x}=\left(b-d-2 \gamma_{0} \bar{F}\right) \frac{\partial \bar{F}}{\partial x},
$$

and the term in parenthesis is negative, unless the equilibrium $(\bar{F}, \bar{B})$ is very close to the Hopf bifurcation (easy to show geometrically by using the so-called isoclines of model (2)). Thus, technological change is associated with an increase of the yield, suddenly interrupted by the collapse of the stock, as already argued long ago (Clark, 1976, 1990) through bioeconomic principles.

\section{The case of polymorphic stocks}

We now consider the case of a single species stock composed of fish with diversified genetic components and focus our attention on the adult body weight as a particular phenotypic trait. Conceptually, we should use a polymorphic model with an extremely high number of morphs (i.e., components of the vector $F$ each associated with different body weights) or, more precisely, study the dynamics of a continuous phenotypic distribution. Since this would be very difficult, we limit our analysis to the case in which the vector $F$ has a limited number of components $F_{1}, \ldots, F_{N}$ corresponding to different body weights $w_{1}, \ldots, w_{N}$. Of course, we must avoid that sexual reproduction introduces new morphs. This is possible through a simple artifact, namely by assuming that newborns from a mother of type $j$ and a father of type $k$ can only be of type $i=1, \ldots, N$, with a bigger fraction $\phi_{j k}^{i}$ when the weight $w_{i}$ is close to the mean of the weights of the parents. The geometric mean $\sqrt{w_{j} w_{k}}$ is conceptually more appropriate than the algebraic one $\left(w_{j}+w_{k}\right) / 2$ since the weight is a positive variable, but the results obtained with the two alternative assumptions are 
almost equivalent. Technically, the fraction $\phi_{j k}^{i}$ is log-normally distributed as

$$
\phi_{j k}^{i}=\frac{\exp \left(-\left(\log \left(w_{i} / \sqrt{w_{j} w_{k}}\right)\right)^{2} / \sigma^{2}\right)}{\sum_{l=1}^{N} \exp \left(-\left(\log \left(w_{l} / \sqrt{w_{j} w_{k}}\right)\right)^{2} / \sigma^{2}\right)},
$$

where $\sigma^{2}$ plays the role of heritability in quantitative genetics (Bulmer, 1980; Falconer, 1989).

The model we use is a simple extension of model (4) where we take into account that reproduction requires the encounter of two mates and that newborns are distributed among the stock components as explained above:

$$
\begin{aligned}
\dot{F}_{i} & =\sum_{j, k=1}^{N} \phi_{j k}^{i} \frac{m b F_{j} F_{k}}{1+m S}-d F_{i}-\sum_{j=1}^{N} \gamma\left(w_{i}, w_{j}\right) F_{i} F_{j} \\
& -E(S) H_{i}\left(F, w, B, B^{\prime}, x\right) B-E(S) H_{i}\left(F, w, B^{\prime}, B, x^{\prime}\right) B^{\prime}, \\
\dot{B} & =\frac{r}{v(x)}\left[p E(S) \sum_{j=1}^{N} w_{j} H_{j}\left(F, w, B, B^{\prime}, x\right)-c_{0}\left(1-\frac{\delta_{e}\left(B+B^{\prime}\right)}{B_{e}+B+B^{\prime}}\right)\right] B-D B, \\
\dot{B}^{\prime} & =\frac{r}{v\left(x^{\prime}\right)}\left[p E(S) \sum_{j=1}^{N} w_{j} H_{j}\left(F, w, B^{\prime}, B, x^{\prime}\right)-c_{0}\left(1-\frac{\delta_{e}\left(B^{\prime}+B\right)}{B_{e}+B^{\prime}+B}\right)\right] B^{\prime}-D B^{\prime} .
\end{aligned}
$$

Here $S=F_{1}+\cdots+F_{N}$ is the total stock abundance,

$$
H_{i}\left(F, w, B, B^{\prime}, x\right)=\frac{a\left(w_{i}, x\right) F_{i} S}{1+\sum_{k=1}^{N} a\left(w_{k}, x\right) h\left(w_{k}, x\right) F_{k} S-\delta_{c}\left(B+B^{\prime}\right) /\left(B_{c}+B+B^{\prime}\right)}
$$

is the functional response specifying the harvest rate (per boat) of fish of type $i$, the new parameter $m$ takes into account the searching and handling of mates, so that the birth rate of $(j, k)$-matings is quadratic at low abundances, while all other parameters are as in model (4).

As for the dependence upon fish size, our assumptions are listed below.

- The intraspecific competition within the stock is best described by the nondimensional competition function $\gamma\left(w_{i}, w_{j}\right) / \gamma\left(w_{i}, w_{i}\right)$ (MacArthur, 1969, 1970) that we fix at 1 in order to describe the simplest case of symmetric competition. We therefore have $\gamma\left(w_{i}, w_{j}\right)=\gamma\left(w_{i}, w_{i}\right)$, i.e., $\gamma$ only depends on its first argument. Moreover, we assume $\gamma\left(w_{i}, w_{i}\right)$ to be $\mathrm{U}$-shaped with a minimum $\gamma_{0}$ at $w_{i}=w_{0}$. 
- The attack rate $a(w, x)$ increases with $w$ and is relatively low up to a threshold size $s$ and relatively high above the threshold. This describes the selectivity of the fishing gears.

- The handling time $h(w, x)$ is increasing with the size $w$ of the fish to be handled, but less than linearly. This takes into account that handling two fishes of equal size requires more effort than handling one fish of double size.

- The specific functions we use are the following:

$$
\begin{aligned}
& \gamma\left(w_{i}, w_{j}\right)=\frac{\gamma_{0}}{2}\left[\left(\frac{w_{i}}{w_{0}}\right)^{2}+\left(\frac{w_{0}}{w_{i}}\right)^{2}\right], \\
& a(w, x)=a_{0} \frac{w^{\alpha}}{s^{\alpha}+w^{\alpha}}\left(1+\frac{\delta_{a} x / x_{a}}{1+x / x_{a}}\right), \quad \alpha>1, \\
& h(w, x)=h_{0}\left(\frac{w}{w_{0}}\right)^{h_{1}}\left(1-\frac{\delta_{h} x / x_{h}}{1+x / x_{h}}\right), \quad h_{1}<1 .
\end{aligned}
$$

Note that in the absence of harvesting, intraspecific competition is the only selective pressure acting on the stock, so that $w_{0}$ represents the fish size to which an unexploited stock would have evolved. In particular, we center our polymorphic distribution around $w_{0}$, by considering a morph in $w_{0}$ itself, plus pairs of morphs in $\rho w_{0}$ and $w_{0} / \rho$ for various values of the size ratio $\rho$ (results are presented for $N=3$, but remain qualitatively similar for $N=5,7, \ldots)$.

As done in the previous section, one can extract from model (5) the corresponding resident model (2), characterize its equilibrium $(\bar{F}(x), \bar{B}(x))$, and derive the corresponding $\mathrm{AD}$ canonical equation (3) (see Appendix B). Also the bifurcation analysis of model (2) is quite similar to the one performed in the case of monomorphic stocks. In particular, the first bifurcation one encounters while increasing the technological level $x$ is still a Hopf bifurcation at which the stable equilibrium $(\bar{F}, \bar{B})$ is substituted by a stable cycle characterized by very long periods of low stock abundance during which the fleet dimension slowly decays. The results of the analysis are not presented because qualitatively very similar to those discussed in the previous section.

The analysis of model (5) and its associated canonical equation allows one to see how the mean weight of the fish

$$
w=\sum_{i=1}^{N} \bar{F}_{i}(x) w_{i} / \bar{S}(x), \quad \bar{S}(x)=\bar{F}_{1}(x)+\cdots+\bar{F}_{N}(x),
$$


varies with technological change. In fact, the curves at constant mean weight $w$ can be drawn in any twodimensional space $\left(q_{i}, x\right)$, as shown in Fig. 4 for the same parameter settings used in Figs. 2 and 3, and the result is fully consistent with property (v) in the Introduction, since for any value of the parameter $q_{i}$ the mean weight is minimum for $x=\bar{x}\left(q_{i}\right)$.

\section{Conclusions and extensions}

We have shown in this paper that the exploitation of fish stocks can entrain in the long run the substantial decline or even the collapse of the stock, difficulties in stock recovery, loss of fishery resilience, and reduction of the mean fish size. The study is carried out for the common case in which the dimension of the harvesting fleet is almost insensitive to the year-to-year fluctuations of the stock. This is a consequence of the typical inertia of fishermen, due to lack of alternatives and/or public or private subsidies, and technically allowed us to use the simple geometric arguments available for the study of slow-fast processes (Rinaldi and Scheffer, 2000). The results are interesting, not only because in agreement with numerous observations, but also because they have been obtained with a simple model of a managed fishery, in which fleet and stock are considered as (slow) predator and (fast) prey, and because the sole cause of evolution is technological innovation. From a formal point of view, the analysis has been performed by means of Adaptive Dynamics (Dieckmann and Law, 1996; Metz et al., 1996; Geritz et al., 1997, 1998; Dercole and Rinaldi, 2008), an approach born in theoretical biology which, however, is used here in the context of technological change.

Although the results are known to all scientists as well as practitioners in the field (Hannesson, 2002; Walters and Martell, 2004), and are qualitatively consistent with those obtained long ago through the principles of bioeconomics (Clark, 1976, 1990), it is fair to stress that the underlying assumptions are different. In fact, in the bioeconomic approach fleet technology either does not evolve or is assumed to increase as an exogenously established fact, while the fishing effort is adjusted to produce economic optimization. By contrast, in the AD approach, technological change is the endogenous result of innovation and competition processes.

The analysis shows that the long-term sustainability of exploited fish stocks can be achieved only if strategic parameters influencing technological change are kept under strict control. This emphasizes the role that fishing agencies can have in protecting exploited fish stocks (Jørgensen et al., 2007). However, it is fair to repeat that the value of the paper is purely conceptual, since our model can hardly be tuned on any 
real fishery. From one side this is a virtue of our study, because being abstract, it shows that the drawn conclusions hold in general. But from the practical side, no directly applicable guidelines for managers can be suggested.

The present study can be extended in various directions but two of them are particularly worth mentioning. The first consists in repeating the analysis with more detailed models in order to derive the most likely consequences of technological change on the age and size structures of the stock, and in particular on its maturation reaction norm (Barot et al., 2004a,b; Ernande and Dieckmann, 2004; Ernande et al., 2004; Olsen et al., 2004; de Roos et al., 2006). The second extension is concerned with the possibility of fleet branching, which, intuitively speaking, could be conceived when fish are polymorphic or have different stages of relevant economic value requiring different fishing technologies. Quite interesting could be, in this context, the study of coevolution (biological and technological) to see if a fishery initially monomorphic (in the fish and in the fleet) can have a first branching in the fish stock entraining a branching in the fleet, and if this process can be repeated, so that through an avalanche of branching pairs the fishery might become highly diversified, both biologically and technologically. A study like this could undoubtedly cast fisheries diversity in a nice theoretical frame.

\section{Acknowledgments}

The authors are grateful to ENS, Paris, and to IIASA, Laxenburg, for supporting the visit of C. P. to Milano and of F. D. to Laxenburg. Thanks are due to Ulf Dieckmann, Bruno Ernande, and to the research group of the IIASA Evolution and Ecology Program for constructive comments on a first draft of this paper. 


\section{References}

2 Allgower, E. L., Georg, K., 1990. Numerical Continuation Methods: An Introduction. Springer-Verlag, Berlin.

4 Alverson, D. L., Freeberg, M. K., Murawski, S. A., Pope, J. G., 1994. A global assessment of fisheries bycatch and discards. FAO Fisheries Technical Paper 339, Food and Agriculture Organization of the United Nations, Rome.

Anderson, C. N. K., Hsieh, C.-H., Sandin, S. A., Hewitt, R., Hollowed, A., Beddington, J., May, R. M., Sugihara, G., 2008. Why fishing magnifies fluctuations in fish abundance. Nature 452, 835-839.

Barot, S., Heino, M., O’Brien, L., Dieckmann, U., 2004a. Estimating reaction norms for age and size at maturation when age at first reproduction is unknown. Evol. Ecol. Res. 6, 659-678.

Barot, S., Heino, M., O’Brien, L., Dieckmann, U., 2004b. Longterm trend in the maturation reaction norm of two cod stocks. Ecol. Applications 14, 1257-1271.

Bellwood, D. R., Hughes, T. P., Folke, C., Nyström, M., 2004. Confronting the coral reef crisis. Nature 429, $827-833$.

Bulmer, M. G., 1980. The Mathematical Theory of Quantitative Genetics. Oxford University Press, New York.

Clark, C. W., 1976. Mathematical Bioeconomics. Wiley, New York.

Clark, C. W., 1990. Mathematical Bioeconomics: The optimal management of renewable resources, 2nd Edition. Wiley, New York.

de Roos, A. M., Boukal, D. S., Persson, L., 2006. Evolutionary regime shifts in age and size at maturation of exploited fish stocks. Proc. R. Soc. Lond. B 273, 1873-1880.

Dercole, F., Rinaldi, S., 2008. Analysis of Evolutionary Processes: The Adaptive Dynamics Approach and its Applications. Princeton University Press, Princeton, NJ.

Dhooge, A., Govaerts, W., Kuznetsov, Yu. A., 2002. MATCONT: A MATLAB package for numerical bifurcation analysis of ODEs. ACM T. Math. Software 29, 141-164. 
Dieckmann, U., Law, R., 1996. The dynamical theory of coevolution: A derivation from stochastic ecological processes. J. Math. Biol. 34, 579-612.

Ellner, S. P., Turchin, P., 1995. Chaos in a noisy world: New methods and evidence from time series analysis. Am. Nat. 145, 343-375.

Ernande, B., Dieckmann, U., 2004. The evolution of phenotypic plasticity in spatially structured environments: Implications of intraspecific competition, plasticity costs and environmental characteristics. J. Evol. Biol. 17, 613-628.

Ernande, B., Dieckmann, U., Heino, M., 2004. Adaptive changes in harvested populations: Plasticity and evolution of age and size at maturation. Proc. R. Soc. Lond. B 271, 415-423.

Falconer, D. S., 1989. Introduction to Quantitative Genetics. Longman, Harlow.

Ferrière, R., Gatto, M., 1993. Chaotic dynamics can result from natural selection. Proc. R. Soc. Lond. B $251,33-38$.

Geritz, S. A. H., Kisdi, E., Meszéna, G., Metz, J. A. J., 1998. Evolutionarily singular strategies and the adaptive growth and branching of the evolutionary tree. Evol. Ecol. 12, 35-57.

Geritz, S. A. H., Metz, J. A. J., Kisdi, E., Meszéna, G., 1997. The dynamics of adaptation and evolutionary branching. Phys. Rev. Lett. 78, 2024-2027.

Hannesson, R., 2002. The economics of fisheries. In: Hart, P. J. B., Reynolds, J. D. (Eds.), Handbook of Fish Biology and Fisheries, vol. 2: fisheries Edition. Blackwell Science, Oxford, UK, pp. 249-269.

Heino, M., 1998. Management of evolving fish stocks. Can. J. Fish. Aquat. Sci. 55, 1971-1982.

Hilborn, R., Walters, C. J., 1992. Quantitative Fisheries Stock Assessment. Chapman \& Hall, New York.

Hutchings, J. A., Reynolds, J. D., 2004. Marine fish population collapses: Consequences for recovery and extinction risk. Bioscience 54, 297-309.

Hutchings, J. A., Reynolds, J. D., 2008. The nature of fisheries- and farming-induced evolution. Mol. Ecol. $17,294-313$. 
Jackson, J. B. C., Kirby, M. X., Berger, W. H., Bjorndal, K. A., Botsford, L. W., Bourque, B. J., Bradbury, R. H., Cooke, R., Erlandson, J., Estes, J. A., Hughes, T. P., Kidwell, S., Lange, C. B., Lenihan, H. S., Pandolfi, J. M., Peterson, C. H., Steneck, R. S., Tegner, M. J., Warner, R. R., 2001. Historical overfishing and the recent collapse of coastal ecosystems. Science 293, 629-638.

Jørgensen, C., Enberg, K., Dunlop, E. S., Arlinghaus, R., Boukal, D. S., Brander, K., Ernande, B., Gårdmark, A., Johnston, F., Matsumura, S., Pardoe, H., Raab, K., Silva, A., Vainikka, A., Dieckmann, U., Heino, M., Rijnsdorp, A. D., 2007. Managing evolving fish stocks. Science 318, 1247-1248.

Kuznetsov, Yu. A., 2004. Elements of Applied Bifurcation Theory, 3rd Edition. Springer-Verlag, Berlin.

Kuznetsov, Yu. A., Muratori, S., Rinaldi, S., 1995. Homoclinic bifurcations in slow-fast second-order systems. Nonlinear Anal. 25, 747-762.

MacArthur, R. H., 1969. Species packing, and what interspecies competition minimizes. Proc. Natl. Acad. Sci. 64, 1369-1371.

MacArthur, R. H., 1970. Species packing and competitive equilibrium for many species. Theor. Popul. Biol. $1,1-11$.

Metz, J. A. J., Geritz, S. A. H., Meszéna, G., Jacobs, F. J. A., van Heerwaarden, J. S., 1996. Adaptive dynamics: A geometrical study of the consequences of nearly faithful reproduction. In: van Strien, S. J., Verduyn Lunel, S. M. (Eds.), Stochastic and Spatial Structures of Dynamical Systems. Elsevier Science, Burlington, MA, pp. 183-231.

Muratori, S., Rinaldi, S., 1989. Catastrophic bifurcations in a second-order dynamical system with applications to acid rain and forest collapse. Appl. Math. Modelling 13, 674-681.

Myers, R., online resource. The Ransom Myers' Stock Recruitment Database. www.mscs.dal.ca/ myers/welcome.html.

Olsen, E. M., Heino, M., Lilly, G. R., Morgan, M. J., Brattey, J., Ernande, B., Dieckmann, U., 2004. Maturation trends indicative of rapid evolution preceded the collapse of northern cod. Nature 428, 932935.

Rinaldi, S., Gragnani, A., 2004. Destabilizing factors in slow-fast systems. Ecol. Modelling 180, 445-460. 
Rinaldi, S., Scheffer, M., 2000. Geometric analysis of ecological models with slow and fast processes. Ecosystems 3, 507-521.

Rosenzweig, M. L., MacArthur, R. H., 1963. Graphical representation and stability conditions of predatorprey interactions. Am. Nat. 97, 209-223.

Salthaug, A., 2001. Adjustment of commercial trawling effort for atlantic cod, Gadus morhua, due to increasing catching efficiency. Fish. Bull. 99, 338-342.

Scheffer, M., Carpenter, S., de Young, B., 2005. Cascading effects of overfishing marine systems. Trends Ecol. Evol. 20, 579-581.

Strogatz, S. H., 1994. Nonlinear Dynamics and Chaos. Addison-Wesley, Reading, MA.

Walters, C. J., Martell, S. J. D., 2004. Fisheries Ecology and Management. Princeton University Press, Princeton, NJ.

Ziman, J. (Ed.), 2000. Technological Innovation as an Evolutionary Process. Cambridge University Press, Cambridge, UK. 


\section{Appendix A}

2 The AD canonical equation (3) corresponding to model (4) reads

$$
\begin{aligned}
\dot{x} & =\left.\bar{B} \frac{\partial}{\partial x^{\prime}}\left(\frac{r}{v\left(x^{\prime}\right)}\left[p w E(\bar{F}) H\left(\bar{F}, 0, \bar{B}, x^{\prime}\right)-c_{0}\left(1-\frac{\delta_{e} \bar{B}}{B_{e}+\bar{B}}\right)\right]-D\right)\right|_{x^{\prime}=x} \\
& =\bar{B} \frac{r}{v(x)}\left[p w E(\bar{F})\left(H_{x}(\bar{F}, 0, \bar{B}, x)-\frac{H(\bar{F}, 0, \bar{B}, x) v_{x}(x)}{v(x)}\right)+c_{0}\left(1-\frac{\delta_{e} \bar{B}}{B_{e}+\bar{B}}\right) \frac{v_{x}(x)}{v(x)}\right],
\end{aligned}
$$

3 where

$$
H_{x}(\bar{F}, 0, \bar{B}, x)=\frac{a_{x}(x) \bar{F}^{2}\left(1-\delta_{c} \bar{B} /\left(B_{c}+\bar{B}\right)\right)-a(x)^{2} h_{x}(x) \bar{F}^{4}}{\left(1+a(x) h(x) \bar{F}^{2}-\delta_{c} \bar{B} /\left(B_{c}+\bar{B}\right)\right)^{2}},
$$

4 the $x$-subscript denotes differentiation with respect to $x$, the term $1 / 2 \mu \sigma^{2}$ in (3) is set to 1 , and the equilib-

5 rium $(\bar{F}, \bar{B})$ is defined by the positive solution of the following two algebraic equations:

$$
\begin{aligned}
& 0=(b-d)-\gamma_{0} \bar{F}-E(\bar{F}) \frac{a(x) \bar{F}}{1+a(x) h(x) \bar{F}^{2}-\delta_{c} \bar{B} /\left(B_{c}+\bar{B}\right)} \bar{B} \\
& 0=\frac{r}{v(x)}\left[p w E(\bar{F}) H(\bar{F}, \bar{B}, 0, x)-c_{0}\left(1-\frac{\delta_{e} \bar{B}}{B_{e}+\bar{B}}\right)\right]-D .
\end{aligned}
$$

6 Equations (A1) form the differential-algebraic system whose equilibrium $(\bar{x}, \bar{F}, \bar{B})$ has been continued with 7 respect to all model parameters in order to produce results like those reported in Figs. 2 and 3. 


\section{Appendix B}

2 The AD canonical equation (3) corresponding to model (5) reads

$$
\begin{aligned}
\dot{x} & =\left.\bar{B} \frac{\partial}{\partial x^{\prime}}\left(\frac{r}{v\left(x^{\prime}\right)}\left[p E(\bar{S}) \sum_{j=1}^{N} w_{j} H_{j}\left(\bar{F}, w, 0, \bar{B}, x^{\prime}\right)-c_{0}\left(1-\frac{\delta_{e} \bar{B}}{B_{e}+\bar{B}}\right)\right]-D\right)\right|_{x^{\prime}=x} \\
& =\bar{B} \frac{r}{v(x)}\left[p E(\bar{S}) \sum_{j=1}^{N} w_{j}\left(H_{j x}(\bar{F}, w, 0, \bar{B}, x)-\frac{H_{j}(\bar{F}, w, 0, \bar{B}, x) v_{x}(x)}{v(x)}\right)+c_{0}\left(1-\frac{\delta_{e} \bar{B}}{B_{e}+\bar{B}}\right) \frac{v_{x}(x)}{v(x)}\right],
\end{aligned}
$$

3 where

$$
\begin{aligned}
H_{i x}(\bar{F}, w, 0, \bar{B}, x) & =\left(a_{x}\left(w_{i}, x\right) \bar{F}_{i} \bar{S}\left(1-\delta_{c} \bar{B} /\left(B_{c}+\bar{B}\right)\right)\right. \\
& +\bar{F}_{i} \bar{S}^{2} \sum_{k=1}^{N}\left(a_{x}\left(w_{i}, x\right) a\left(w_{k}, x\right)-a\left(w_{i}, x\right) a_{x}\left(w_{k}, x\right)\right) h\left(w_{k}, x\right) \bar{F}_{k} \\
& \left.-a\left(w_{i}, x\right) \bar{F}_{i} \bar{S}^{2} \sum_{k=1}^{N} a\left(w_{k}, x\right) h_{x}\left(w_{k}, x\right) \bar{F}_{k}\right) \\
& /\left(1+\sum_{k=1}^{N} a\left(w_{k}, x\right) h\left(w_{k}, x\right) \bar{F}_{k} \bar{S}-\delta_{c} \bar{B} /\left(B_{c}+\bar{B}\right)\right)^{2},
\end{aligned}
$$

${ }_{4} \bar{S}=\bar{F}_{1}+\cdots+\bar{F}_{N}$, the $x$-subscript denotes differentiation with respect to $x$, the term $1 / 2 \mu \sigma^{2}$ in (3) is set to 51 , and the equilibrium $\left(\bar{F}_{i}, \bar{B}\right), i=1, \ldots, N$, is defined by the positive solution of the following algebraic 6 equations:

$$
\begin{aligned}
& 0=\sum_{j, k=1}^{N} \phi_{j k}^{i} \frac{m b \bar{F}_{j} \bar{F}_{k}}{1+m \bar{S}}-d \bar{F}_{i}-\sum_{j=1}^{N} \gamma\left(w_{i}, w_{j}\right) \bar{F}_{i} \bar{F}_{j}-E(\bar{S}) H_{i}(\bar{F}, w, \bar{B}, 0, x) \bar{B}, \\
& 0=\frac{r}{v(x)}\left[p E(\bar{S}) \sum_{j=1}^{N} w_{j} H_{j}(\bar{F}, w, \bar{B}, 0, x)-c_{0}\left(1-\frac{\delta_{e} \bar{B}}{B_{e}+\bar{B}}\right)\right]-D .
\end{aligned}
$$

7 Equations (B1) form the differential-algebraic system whose equilibrium $\left(\bar{x}, \bar{F}_{i}, \bar{B}\right), i=1, \ldots, N$, has been 8 continued with respect to all model parameters in order to produce results like those reported in Fig. 4. 


\section{Figure captions}

\section{Figure 1}

4 Trajectories of system (2) for the following parameter values: $b=2, d=1, \gamma_{0}=1, r=0.1, p=1$, ${ }_{5} w=1, c_{0}=0.2, \delta_{e}=0.2, B_{e}=10, D=0.00799, \delta_{c}=0.2, B_{c}=10, F_{0}=0.01, e=4, v_{0}=10$,

$6 v_{1}=2, x_{v}=10, a_{0}=1000, \delta_{a}=0.5, x_{a}=10, h_{0}=1, \delta_{h}=0.5, x_{h}=10$. Double arrows indicate fast 7 motion. (a) $0.02=x<x^{*} \simeq 0.0408$, the equilibrium $(\bar{F}, \bar{B})$ (filled circle) is stable. (b) $0.05=x>x^{*}$, 8 the equilibrium $(\bar{F}, \bar{B})$ (empty circle) is unstable and surrounded by a stable limit cycle (thick trajectory).

\section{Figure 2} canonical equation (3) as a function of attack rate $a_{0}$ (log-scale) (panel a) and protectionism $F_{0}$ (panel b) (in 3 both cases the parameter dependence of $\bar{x}$ is very weak). Arrows indicate direction of technological change. Technological levels between the two branches of the Hopf bifurcation curve (dotted segments) give rise to limit cycles in model (2). Other parameter values as in Fig. 1.

17 Figure 3

Stock abundance $\bar{F}$ (low=white, large=black) for the two cases examined in Fig. 2.

Figure 4

Mean weight $w$ of the fish (low=white, large=black) in the same spaces considered in Figs. 2 and 3. 

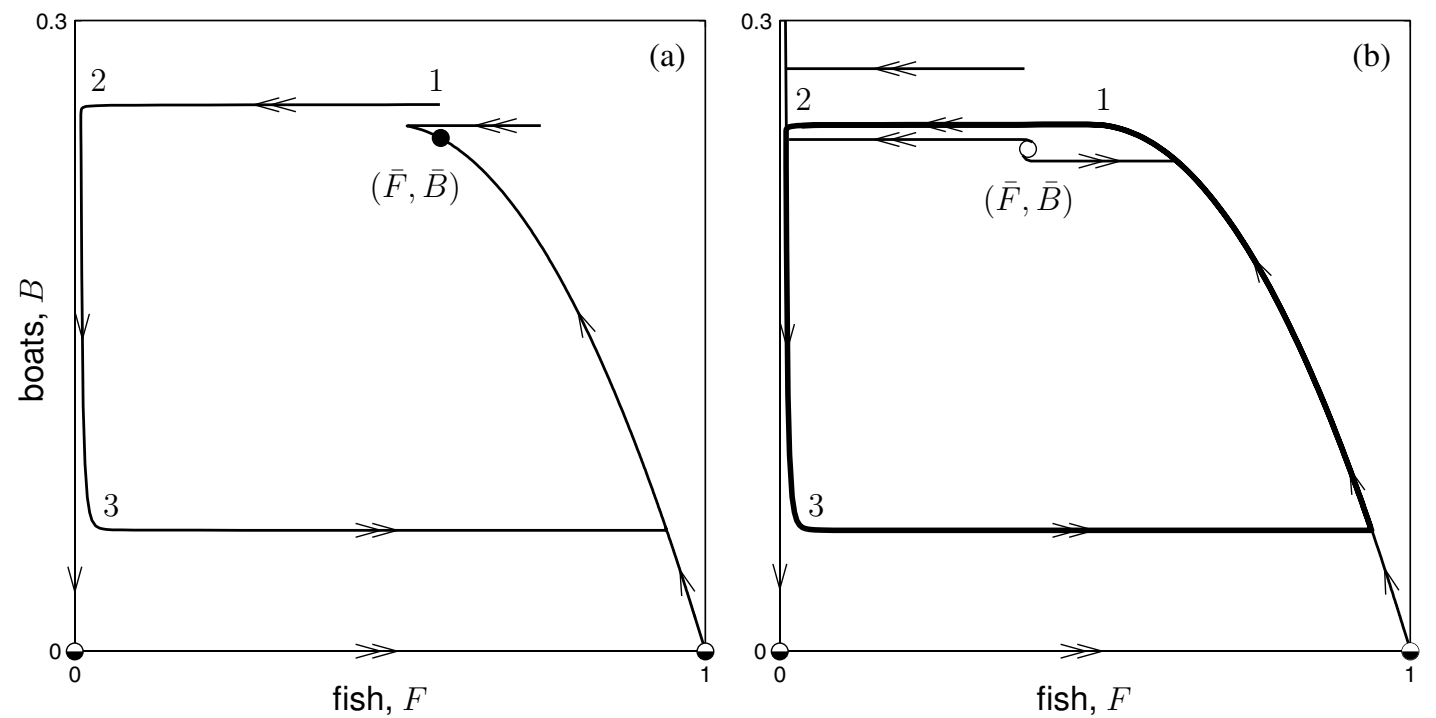

Figure 1 

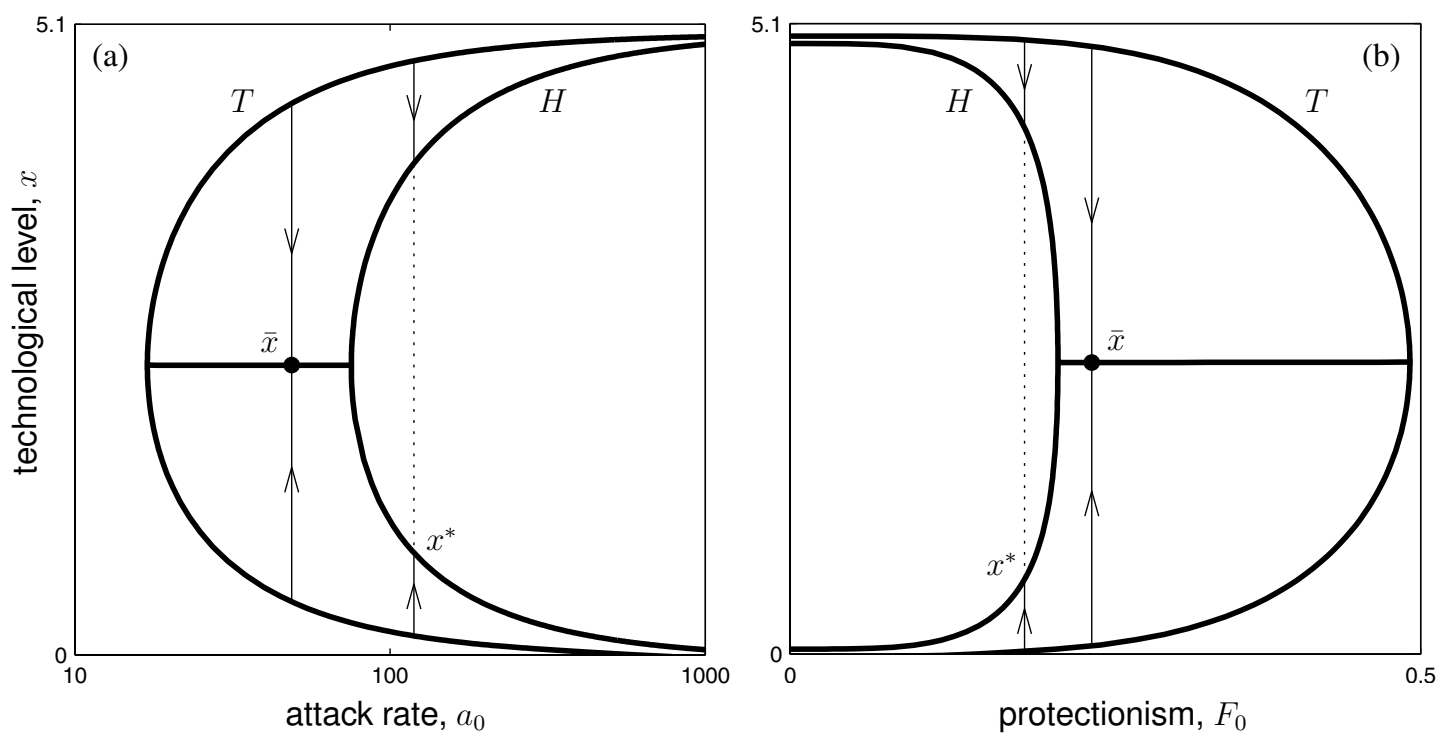

Figure 2 

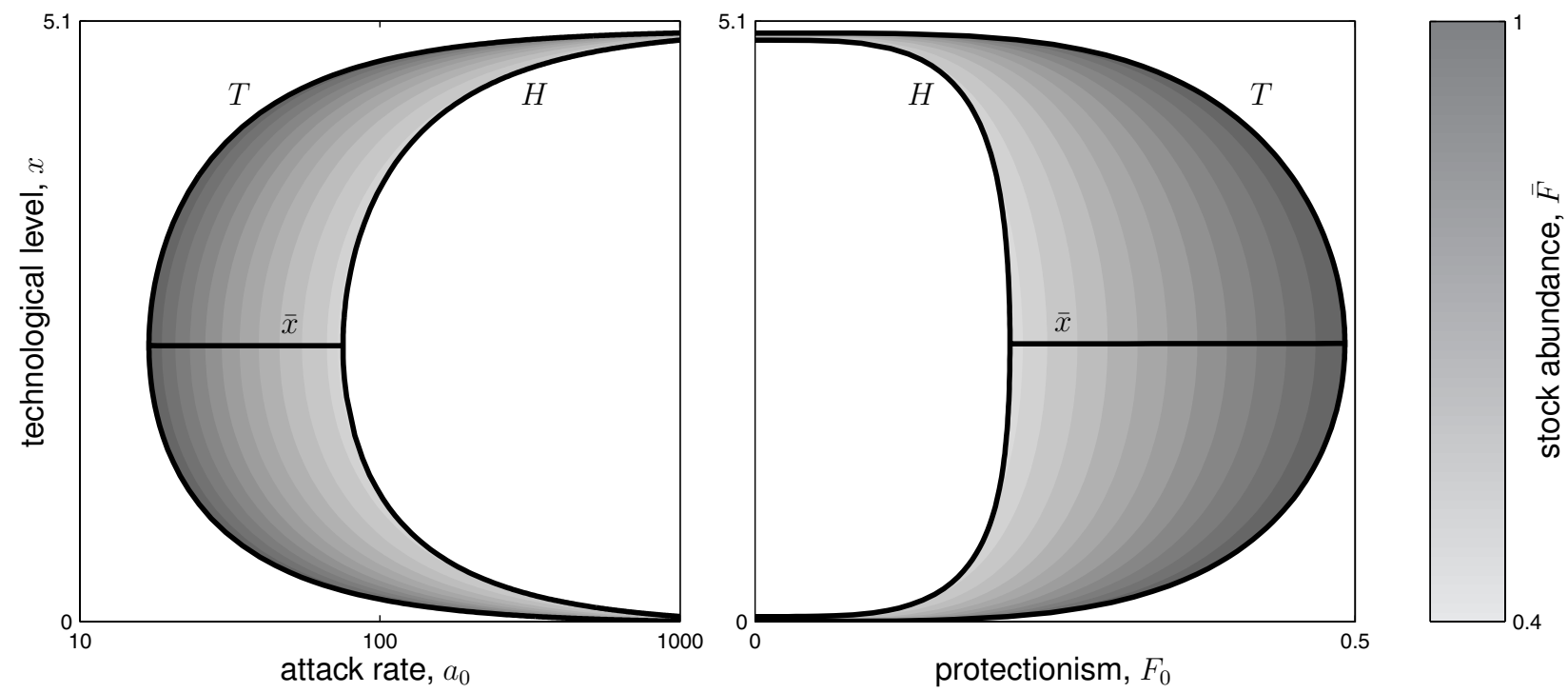

Figure 3 

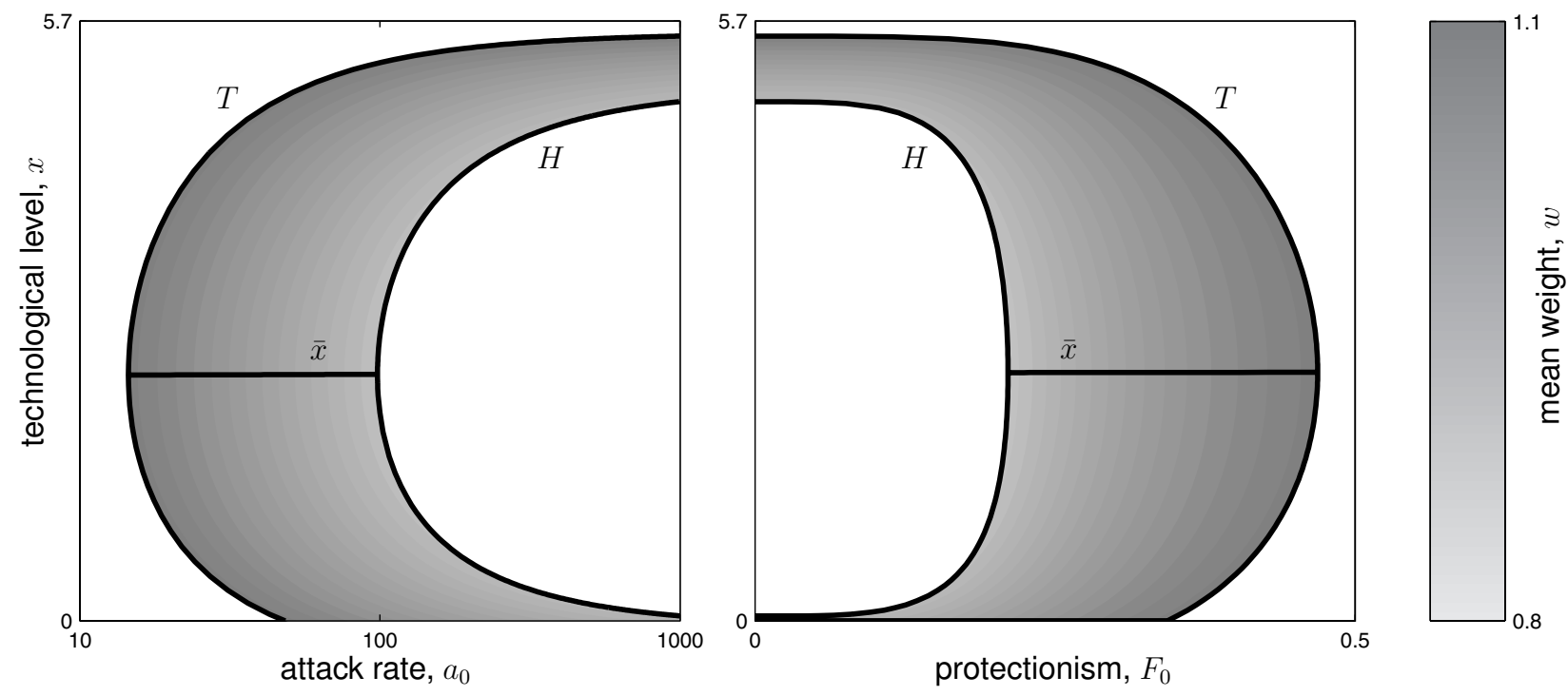

Figure 4 